\title{
Separación o divorcio de los padres. Consecuencias en los hijos y recomendaciones a los padres y pediatras
}

\author{
Parents separation or divorce. Potential effects on children and recommendations \\ to parents and pediatricians
}

\author{
Arturo Roizblatt S. ${ }^{a}$, Valentina M. Leiva F. ${ }^{b}$, Ana Margarita Maida S. ${ }^{c}$
}

aédico Psiquiatra. Departamento de Psiquiatría Campus Oriente Facultad de Medicina Universidad de Chile

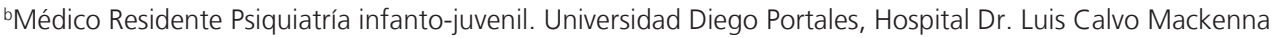

cMédico Psiquiatra infanto-juvenil, Terapeuta familiar, Hospital Dr. Luis Calvo Mackenna

Recibido el 6 de julio de 2017; aceptado el 12 de octubre de 2017

\section{Resumen}

El divorcio o separación de una pareja con hijos pone fin a la convivencia de ambos padres en conjunto con sus hijos. Puede haber múltiples causas para un divorcio y tanto la literatura como la experiencia de los autores trabajando con familias en esta situación, dan cuenta de algunas consecuencias a corto y largo plazo para los niños, especialmente en aquellos casos en que el divorcio ha sido conflictivo. En estos casos, se ha observado mayor riesgo de presentar trastornos de conducta, bajo rendimiento escolar y abuso de sustancias. Por otra parte puede tener consecuencias en la vida adulta, presentando mayor riesgo de patologías psiquiátricas o dificultades en las relaciones interpersonales si no se realiza una intervención que aborde algunas situaciones potencialmente traumáticas para los niños. Los pediatras se encuentran en una relación privilegiada con respecto a los niños y sus familias para detectar signos de discordia parental y alteración de la salud mental infantil. Las conductas que se manifiesten en los niños a consecuencia del divorcio y el conflicto parental van a depender de la edad y etapa de desarrollo del niño, siendo importante reconocerlas para poder intervenir de forma adecuada. El presente artículo propone directrices para guiar a los padres, ya que con un adecuado manejo de las situaciones de conflicto que ocurren alrededor del divorcio es posible prevenir algunas de las consecuencias que éste puede tener en los hijos.
Palabras clave: Divorcio; conflicto familiar; padres; relaciones padres-hijo; psicología infantil; familia

\section{Keywords:}

Divorce; family conflict; parents; parent-child relationships; child psychology; family 
consequences in adult life with higher risk of psychiatric pathologies or difficulties in interpersonal relationships, if an intervention that addresses some potentially traumatic situations for children is not done. Pediatricians are in a privileged relationship with children and their families in order to detect signs of parental discord and altered mental health in children. Children behaviors as a result of divorce and parental conflict will depend on the age of the child and stage of development, and it is important to recognize them in order to intervene properly. This article proposes some guidelines for parents. Good management of high conflict situations related to divorce may prevent some of the consequences that these can have on children.

El divorcio como proceso que da término a la unión conyugal está legislado prácticamente en todo el mundo. El último país de Latinoamérica en legislarlo fue Chile, en el año 2004. En Estados Unidos la tasa de divorcio y anulaciones es de un 3,2 por 1.000 habitantes ${ }^{1}$. En Chile la tasa de divorcio correspondía a un 0,1 por 1000 habitantes en el año 2009, llegando a un 2,7 en $2014^{2-4}$. Una investigación realizada por la Organización para la Cooperación y el Desarrollo Económicos (OCDE) mostró que en general ha existido un aumento de las tasas de divorcio acompañado de una disminución de las tasas de matrimonio en los países pertenecientes a esta organización ${ }^{5}$.

En el presente artículo se hablará indistintamente de divorcio y separación de la pareja, ya que cuando hay hijos, finaliza la convivencia común entre padres e hijos. El divorcio puede ocurrir en una variada gama de situaciones; desde aquella en que los padres deciden divorciarse habiendo procurado mantener hasta ese momento a los hijos fuera del conflicto conyugal; hasta aquel en que los hijos han estado intensamente involucrados y expuestos al conflicto que precede a la separación e incluso a la que continúa después de ésta.

Los niños criados en un hogar donde existe armonía parental tienen mejor salud física y mental ${ }^{6}$. Por el contrario, la discordia parental los afecta profundamente y produce numerosas consecuencias en distintas esferas de su vida ${ }^{7}$.

Como mencionan Cohen \& $\mathrm{Carol}^{8}$, el pediatra, así como el médico de familia, en cierto modo equivalen al antiguo "médico de cabecera". Está atento no solo a los problemas físicos que afectan a los niños durante su desarrollo, sino también a los estresores que pueden estar presentes. En el caso de notar que en la familia existe discordia parental, una de sus tareas podría ser preguntar por ello, hablar con los padres sobre cómo esta afecta al niño y referirlos a una terapia de pareja o familiar, según sea el caso y la disposición de la familia.

El pediatra también puede realizar una guía anticipatoria con los padres que enfrentan una separación o divorcio. Le es posible mantener una relación con ambos padres e insistirles que, más allá de la situación legal, lo que le interesa y preocupa es la calidad de la relación que ambos mantengan con sus hijos ${ }^{8}$.
En el presente artículo se analizan algunas de las consecuencias que el divorcio o separación puede traer a los hijos, con el objetivo de entregar recomendaciones a los pediatras para ayudarlos a guiar a los padres a entender algunas reacciones y cómo proteger el bienestar de los niños.

\section{Consecuencias en los hijos luego del divorcio o separación de los padres}

El divorcio produce alteración del bienestar del niño si es contencioso y si se asocia a empeoramiento de la situación económica, mal funcionamiento conductual y emocional de los padres, crianza ineficaz, pérdida de contacto con uno de los padres, persistencia de conflictos entre los cónyuges y ausencia de acuerdos en la crianza ${ }^{9,10}$. Las alteraciones de la parentalidad que se presentan en divorcios contenciosos pueden ser estresores capaces de generar mala adaptación en respuestas neuropsicobiológicas, generando deterioro en la salud física y mental infantil ${ }^{7}$.

En general, la mayoría de las dificultades de adaptación en los niños después de la separación o divorcio, se resuelve dentro de 2-3 años y entre 3-5 años luego del segundo matrimonio, si lo hay ${ }^{11}$. El posible efecto traumático del divorcio va disminuyendo a medida que pasa el tiempo ${ }^{12}$, y muy especialmente si los padres logran acuerdos en torno a la crianza. En algunos casos, sin embargo, se ha observado que los problemas emocionales pueden aumentar con la edad y pueden no expresarse hasta la adolescencia tardía o adultez ${ }^{13}$.

La relación entre género y los efectos del divorcio conflictivo es un factor ampliamente discutido. Las investigaciones han sido inconsistentes o no han podido establecer la relación entre el género y el divorcio de los padres $^{14}$.

En una revisión se observó que los niños eran afectados más negativamente que las niñas ${ }^{15}$. Estos niños tenían un mayor historial de consultas psiquiátricas durante la infancia y adolescencia y presentaban más síntomas de ansiedad de separación ${ }^{16}$, más miedos escolare $^{17}$ y más problemas de conducta ${ }^{18}$. Otros estudios, por el contrario, han observado que las niñas son 
más afectadas negativamente que los niños, pudiendo explicarse por el rol de contención emocional que a veces deben ofrecer a alguno de los padres ${ }^{19}$.

Esta inconsistencia podría sugerir que tanto hombres como mujeres son afectados, pero de distinta manera ${ }^{10}$.

Se ha observado que el divorcio de los padres aumenta el riesgo de consumo de alcohol en los adolescentes, lo que puede estar relacionado, entre varios factores, a un menor control parental o a una peor situación socioeconómica familiar ${ }^{20}$.

Muchos niños experimentan sentimientos de pérdida cuando uno de los padres deja el hogar de la familia, pero algunos sienten alivio ${ }^{21}$. Este alivio sería explicable si un ambiente hostil y de violencia se interrumpe al ocurrir la separación de la pareja. Cabe destacar que el divorcio es un proceso y no solo un evento, al menos para comprender los efectos en los niños ${ }^{22}$.

Los niños tienen mayor probabilidad de presentar problemas psicológicos cuando existe una relación conflictiva entre los padres antes, durante o después del divorcio ${ }^{23}$. Generalmente el conflicto interparental es mayor en el período inmediato al divorcio y se espera vaya disminuyendo con el tiempo, sin embargo hasta un $44 \%$ de las familias divorciadas permanecen en conflicto por períodos prolongados que van más allá de 3 años $^{24}$, e incluso pueden terminar comprendiendo toda la vida. Este conflicto afecta la calidad de vida de los niños ${ }^{25}$, genera trastornos de ansiedad y alteración en el rendimiento académico ${ }^{26}$.

\section{Consecuencias a largo plazo en el plano afectivo de la discordia parental y divorcio}

El divorcio de los padres puede cambiar la mirada de los hijos en torno al amor y las relaciones, percibiendo que estas son inestables y susceptibles de decepciones $^{27}$. Además, un elevado nivel de conflicto parental puede transmitirse como un modelo negativo para el manejo de las relaciones, desarrollando peores habilidades de enfrentamiento a los conflictos de pareja $^{28}$. Una mayor exposición al conflicto parental en la niñez, se asocia, en la adolescencia y adultez, con parejas que terminan su relación más rápido, tienen relaciones insatisfactorias y mayor violencia dentro de la pareja ${ }^{22,29-31}$.

El conflicto parental tiene mayor impacto en las conductas sexuales de riesgo en los hijos que el divorcio de los padres ${ }^{32}$. Se ha observado que las hijas adolescentes tienen una visión menos positiva de las relaciones amorosas, menos probabilidades de involucrarse en ellas, y si lo hacen, son menos estables ${ }^{28}$. Wallerstein y Corbin encontraron que una relación padre-hija deficiente se asoció con un ajuste social de- ficiente en las hijas (hasta diez años postseparación) y que durante la adolescencia estas niñas tenían mayor probabilidad de una actividad sexual precoz y promiscua $^{33}$. También se ha observado que cuando las madres son capaces de apoyar el surgimiento de los intereses románticos, las hijas pueden desarrollar aptitudes sentimentales adecuadas ${ }^{28}$.

\section{Cuidados y residencia primaria}

Se denomina «residencia primaria», a aquella donde el niño vive más tiempo. En Chile, habitualmente, este es el hogar materno. Sin embargo con el fin de facilitar el tránsito entre un tipo de familia biparental a uno uniparental, ambos padres pueden participar con los mismos derechos y deberes en la crianza y educación de sus hijos, que es lo que se pretende a través del concepto de "co-custodia" 34,35 o custodia compartida.

Este sistema es aquel en que el padre y madre comparten la autoridad y responsabilidad de la toma de decisiones respecto de sus hijos y ambos se ven a sí mismos como igualmente significativos en la vida de ellos. Se considera un sistema favorable para evitar las consecuencias negativas que pueda tener el divorcio ${ }^{36}$. Se ha demostrado que un mayor nivel de apoyo y control por parte del padre no residente, luego de un divorcio, tiene consecuencias positivas en el bienestar de los niños ${ }^{37} \mathrm{e}$ influye positivamente en la autoestima de esos hijos ${ }^{38}$.

También se ha observado que los adolescentes que tienen custodia compartida luego de un divorcio presentan significativamente menor riesgo de abuso de alcohol que los que no la tienen ${ }^{39}$, además existen menores quejas psicológicas por parte de los adolescentes que tienen custodia física compartida en comparación con los que residen con un solo padre ${ }^{40}$.

A pesar de esto, lo más frecuente es que se dé la «custodia única», aquella en la que uno de los padres ejerce la responsabilidad «total» del niño. Aun así, a través del tiempo se pueden dar cambios, como por ejemplo en los adolescentes, que al sentirse "controlados" por el progenitor con quien viven expresen deseos de irse a vivir con el otro, con quien tienen fantasías de que serán «libres y felices». De hecho, la mayoría de los adolescentes en esta situación, al poco tiempo después de cambiarse de casa, deciden regresar a su residencia primaria.

Otro tipo de acuerdo, aunque más infrecuente aún por lo difícil, es que los hijos vivan en un hogar y sean los padres quienes vayan turnándose para vivir en esa casa, lo que exigirá mayor fluidez en la calidad de la comunicación entre ellos y a la vez, reglas sumamente claras.

En general, tanto los estudios de hijos de matrimo- 
nios divorciados como nuestra propia experiencia en este campo, apuntan a que la adaptación social de los niños depende de la permanencia o no del conflicto interparental.

En relación a la presencia del padre no custodio en la vida de los niños, un seguimiento a 8 años mostró que los jóvenes expuestos a altos niveles de conflicto interparental presentan peor adaptación psicosocial, aun cuando ese padre siga involucrado activamente en sus vidas ${ }^{24}$. En el mismo estudio se apreció que los hijos que no tuvieron contacto personal con el padre no custodio, no tuvieron peores resultados que los que mantuvieron contacto cercano pero con alto nivel de conflicto, sugiriendo que una constante relación padre-hijo no necesariamente, por sí misma, mitiga las consecuencias de una relación interparental conflictiva $^{24}$. Algunos estudios sugieren que si el niño mantiene contacto cercano y satisfactorio con el padre que no custodia, aún en presencia de conflicto entre la pareja, los efectos del divorcio pueden no ser perjudiciales ${ }^{9}$. Otro estudio muestra que la migración de un padre a una localidad lejana podría incluso proteger a los hijos/as de conflictos interparentales y/o de disrupciones persistentes si el conflicto parental es intenso ${ }^{41}$.

\section{Rol del pediatra en casos de divorcio o separación}

Los pediatras deben estar preparados para lidiar con múltiples crisis en las vidas de las familias que atienden, entre ellas, la separación y el divorcio suelen ser temas por las que los padres les consultan.

En general, el pediatra puede decirle a los padres que el bajo nivel de conflicto interparental es considerado un factor protector y se ha demostrado que una buena relación con los padres puede ser una defensa para las consecuencias indeseables de un divorcio ${ }^{20}$. Hay que enfatizar que los efectos del divorcio se dan no por la separación en sí, sino debido a la forma inadecuada de los padres de enfrentarla, por lo tanto, se pueden prevenir los efectos adversos y se considera que los pediatras pueden desempeñar un rol fundamental con el fin de educar en ese sentido.

Los niños pequeños no son capaces de entender las circunstancias ambientales, pero sí perciben los cambios de su entorno, de sus cuidadores y/o rutinas. Ellos necesitan estabilidad en la rutina diaria y suficiente contacto con un cuidador principal para desarrollar un apego seguro. El apego temprano seguro será la base para el desarrollo de futuras relaciones y los conflictos de alta intensidad están vinculados con el desarrollo de estilos de apego inseguro y desorgani$z^{2}{ }^{34}$. En situaciones de estrés externo, los lactantes pueden manifestar signos de irritabilidad, retraimiento o apatía, cambio en los patrones del sueño o el apetito $^{8}$.

La recomendación en este caso sería que el padre no custodio lo visite en su hogar, mientras el niño pernocta con el padre custodio. Evitar cambios en su rutina. Ponerse de acuerdo en hitos fundamentales como el horario de alimentación, sueño, juegos.

En los pre-escolares la regresión es una reacción común. Se puede proteger a los niños en este proceso entregando mayor atención uno a uno e informando de forma adecuada a su nivel de desarrollo cómo será la rutina familiar y cómo continuará la relación con sus padres ${ }^{27}$.

Erik Erikson ${ }^{42}$ señala que entre los tres y cuatro años de edad comienza a desarrollarse en los niños el sentido de lo moral, lo bueno y lo malo, que lleva aparejado el concepto de culpa. Esta culpa, sumada al egocentrismo propio de esa edad, hace que muchas veces se acusen a sí mismos de los problemas de sus padres y del divorcio.

Las recomendaciones en esta etapa son repetir que ellos no tienen culpa de la separación, que esta es cuestión de los adultos y que son los padres quienes resolverán de la mejor forma cómo va a continuar la familia.

También a esta edad es necesario enfatizar en la continuidad de las rutinas. El niño puede empezar a pernoctar con el padre no custodio, siempre y cuando esto sea bien preparado por ambos padres. Tal vez empezar por unas pocas horas, hasta completar un tiempo más prolongado.

Los niños de 6 a 8 años pueden interpretar que la separación de sus padres es el colapso de todo el medio que los rodea y protege. Es común también, que los niños manifiesten sentimientos de culpa y deslealtad, con uno y otro padre, yendo contentos a alojar con su padre no custodio, y al mismo tiempo culpables por dejar al progenitor con quien viven, situación que puede invertirse al momento de regresar al hogar primario.

Los escolares pueden culparse a ellos mismos por los problemas de pareja y eventual separación siendo frecuentes las fantasías de reencuentro y armonía entre sus padres. Pueden presentar cambios de ánimo, especialmente mayor tristeza y enojo ${ }^{8,27}$.

En caso de producirse un divorcio, los padres pueden ayudar a sus hijos en esta edad, fomentando confianza y confirmando la estabilidad de esta nueva y distinta organización familiar. Necesitan recordatorios que el divorcio es definitivo y que ellos no tienen la culpa. A toda edad, pero muy especialmente en esta, se deben mantener límites claros y exponer las consecuencias en caso de violación de las reglas, lo que idealmente debería ser expresado de forma concordante por ambos padres. También es importante proporcio- 
nar un espacio para que los niños expresen sus sentimientos abiertamente ${ }^{27,34}$

Los adolescentes pueden comprender mejor las situaciones complejas y abstractas involucradas en la discordia parental y el divorcio, pero igualmente pueden tener dificultad en aceptarlo. Pueden asumir responsabilidades excesivas y estar preocupados por asuntos que competen a los adultos, a veces en situaciones en que los propios padres parecen estar viviendo una "segunda adolescencia", con la consiguiente confusión en las reglas que rigen la disciplina familiar. Estos adolescentes pueden sentirse sin límites, lo que les puede provocar inseguridad o llevar a conductas poco saludables. Algunos estudios han demostrado que los adolescentes que viven en familias de matrimonios divorciados tienen mayor riesgo de presentar problemas externalizantes (uso de alcohol, violación de reglas) e internalizantes (depresión, ansiedad, retraimiento de su grupo familiar y amigos) en comparación con adolescentes de familias no divorciadas $^{27}$.

En esta etapa, los padres debieran ayudar a sus hijos delimitando hasta dónde pueden compartir sus problemas, y deben mantener en intimidad aquellas situaciones para cuya cabal comprensión un adolescente aún no está preparado, como asuntos legales, sexuales, de pareja o económicos ${ }^{43}$.

En todas las fases, pre, peri y postseparación, es necesario dar información adecuada a los hijos. Al respecto, pueden hacerse las siguientes consideraciones generales $^{44}$ :

1. Hay que manifestarles claramente que el matrimonio se separará, dando los motivos que sean más comprensibles para su edad; explicarles que ellos no causaron ni motivaron la separación; manifestarles que ellos no podrían haber influido ni impedido la separación, explicarles que ésta es una situación del matrimonio y que nada tiene que ver con ellos (en la causa) y que tampoco podrán hacer nada para una eventual reconciliación.

2. Enfatizar que ambos siguen siendo padres a pesar de que se acabe el matrimonio; decirles que no tienen que elegir entre uno de los dos padres; hacerles saber que sus padres comprenden que tengan dudas, que siempre estarán dispuestos a escucharlos y a responderles.

3. Hacerles saber que es normal que tengan rabia, pena o confusión y que esto no sólo pueden hablarlo dentro de la familia, sino también fuera de ella y manifestarles claramente que los padres se amaban cuando ellos fueron gestados y que son producto de ese acto amoroso.

Las siguientes sugerencias respecto al acceso a los hijos dependen de cada caso:
- Es recomendable que los encuentros del padre o madre con los hijos con quienes no vive, no se realicen en ambientes artificiales y dedicados sólo a la diversión; las actividades a las que den lugar deben estar ligadas a lo cotidiano: llevarlos o traerlos del colegio, hacer tareas juntos, etcétera.

- Dependiendo del tipo de relación, se puede explorar la conveniencia de que niños menores de tres años duerman fuera de su hogar. Si lo hacen, el progenitor el que vive con niño debe ser ubicable fácilmente, por si el niño desea retornar al hogar primario o contactarse con su padre custodio.

- En todas las otras edades, las salidas deben ser predecibles, estables en su frecuencia, informando la hora exacta a la que se les pasará a buscar y a dejar, y que el padre o madre la cumpla en forma rigurosa para infundir confianza en los acuerdos parentales.

- Entre los diez y catorce años y la adolescencia, es esperable, a veces, que el niño desee distanciar los contactos con el progenitor no custodio. En estos casos, las estadías fuera de la casa deben ser según su deseo, ya que la relación con sus amistades es lo que más le interesa.

- Ya sea porque son hombres o mujeres o por sus diferentes edades o intereses, es conveniente considerar algunos encuentros con algunos hijos o hijas, y no con todos a la vez, sin que esto se traduzca en un desbalance que muestre algún grado de favoritismo.

Lo expuesto no son reglas rígidas y si de hecho se ha llegado a acuerdos distintos a los que aquí se han descrito y han resultado satisfactorios para padres e hijos, sería preferible no modificarlos ${ }^{44}$.

En respuesta a los aumentos de las tasas de divorcio, muchos países han adoptado un programa educacional obligatorio al momento en que se solicita el divorcio. Estos programas generalmente son breves y didácticos, focalizándose en cómo ayudar a los niños a enfrentar la situación durante e inmediatamente después del proceso de divorcio. Un grupo de investigación en Australia ${ }^{45}$ ha implementado un programa de mediación que incluye a los niños y se ha visto que tiene un efecto en reducir los niveles de conflicto y un mejor manejo de éstos. También se proponen nuevos diseños para aplicar en los tribunales con el fin de realizar un divorcio colaborativo en vez un enfoque confrontacional ${ }^{46}$.

\section{Conclusión}

La situación de separación y divorcio en la familia produce cambios intensos y extensos en diversas 
dimensiones. La decisión de cómo se vivirá en familia después de un divorcio, es un tema que amerita una profunda discusión y un profundo compromiso para continuar ejerciendo la coparentalidad.

Hemos procurado entregar algunas directrices a los pediatras para ayudar a los padres inmersos en el conflicto a privilegiar el bienestar de sus hijos, de tal modo que los niños pueden transitar por esta experiencia sin efectos adversos, y por el contrario, aprender que es posible lograr soluciones a problemas de difícil manejo.

\section{Conflicto de intereses}

Los autores declaran no tener conflicto de intereses.

\section{Referencias}

1. National Marriage and Divorce Rate Trends, CDC/NCHS National Vital Statistics System. 2014 Disponible en: https://www.cdc.gov/nchs/nvss/marriage_ divorce_tables.htm. Última visita 01-1216.

2. Divorce and crude divorce rates. United Nations Statistical Division (UNSTAT) 2011. http://unstats.un.org/unsd/ demographic/products/dyb/dyb2011/ Table25.pdf. Última visita: 01-06-2017.

3. Informe anual de justicia, Instituto nacional de estadística, Chile. 2014. Disponible en: http://www.ine.cl/docs/ default-source/publicaciones/2015/ justicia_2014.pdf?sfvrsn=6. Última visita: 01-12-16

4. Estadísticas vitales anuario 2014, Instituto Nacional de estadística, Chile. Disponible en: http://www.ine.cl/canales/ menu/publicaciones/calendario_de_ publicaciones/pdf/completa_vitales_2014. pdf. Última visita: 01-12-16.

5. OECD Family Database, SF3.1: Marriage and divorce rates. Disponible en: https:// www.oecd.org/els/family/SF_3_1_ Marriage_and_divorce_rates.pdf. Última visita: 01-12-16

6. Anderson J. The impact of family structure on the health of children: Effects of divorce. Linacre Q. 2014; 81(4):378-87.

7. Nunes-Costa R, Lamela D, Figueiredo B. Psychosocial adjustment and physical health in children of divorce. J Pediatr (Rio J). 2009;85(5):385-96.

8. Cohen G, Weitzman C. Helping Children and Families Deal With Divorce and Separation. Pediatrics. 2016;138(6):e20163020.

9. Fabricius W, Luecken L. Postdivorce living arrangements, parent conflict, and long-term physical health correlates for children of divorce. J. Fam. Psychol. 2007; 21:195-205

10. Lacey R, Bartley M, Pikhart H, Stafford M, Cable N. Parental separation and adult psychological distress: an investigation of material and relational mechanisms. BMC Public Health. 2014;14:272.

11. Hetherington E. Divorce and the adjustment of children. Pediatr Rev. 2005;26(5):163-9.
12. Reiter F, Hjörleifsson S, Breidablik $\mathrm{H}$, Meland E. Impact of divorce and loss of parental contact on health complaints among adolescents. J Public Health (Oxf). 2013;35(2):278-85.

13. Huurre T, Junkkari H, Aro H. Long-term psychosocial effects of parental divorce: a follow-up study from adolescence to adulthood. Eur Arch Psychiatry Clin Neurosci. 2006;256(4):256-63.

14. Hetherington $M$. The Influence of Conflict, Marital Problem Solving and Parenting on Children's Adjustment in Nondivorced, Divorced and Remarried Families. En: Alison Stewart C. y Dunn J. eds. Families Count, Cambridge; Cambridge University Press, 2006; 20337.

15. Nederhof E, Belsky J, Ormel J, Oldehinkel A. Effects of divorce on Dutch boys'and girls'externalizing behavior in Gene $\mathrm{x}$ Environmenent perspective: Diathesis stress or differential susceptibility in the Dutch Tracking Adolescents Individual Lives survey study? Dev Psychopathol. 2012;24:929-39.

16. Orgilés M, Espada J, Méndez X. Trastorno de ansiedad por separación en hijos de padres divorciados. Psicothema. 2010;20:383-8

17. Orgilés M, Espada J, Méndez X, et al. Miedos escolares en hijos de padres divorciados y no divorciados. Psicothema. 2008;8:693-703.

18. Cantón J, Cortés M, Justicia M. Las consecuencias del divorcio en los hijos. Psicopatol clín leg forense. 2002;2(3):4766.

19. Angarne-Lindberg T, Wadsby M. Fifteen years after parental divorce: Mental health and experienced life-events. Nord J Psychiatry 2009;63(1):32-43.

20. Tomcikova Z, Madarasova G, Van Dijk J, Reijneveld S. Characteristics of adolescent excessive drinkers compared with consumers and abstainers. Drug Alcohol Rev. 2011;30(2):157-65.

21. Grant J, Waldron M, Sartor C, et al. Parental Separation and Offspring Alcohol Involvement: Findings from Offspring of Alcoholic and Drug Dependent Twin Fathers. Alcohol Clin Exp Res. 2015;39:1166-73.

22. Amato P. Research on divorce:
Continuing trends and new developments. J Marriage Fam. 2010;72:650-66.

23. Grych J. Interparental conflict as a risk factor for child maladjustment: implications for the development of prevention programs. Fam Ct Rev. 2005;43:97-108.

24. Modecki K, Hagan J, Sandler I, Wolchik S. Latent Profiles of Nonresidential Father Engagement Six Years After Divorce Predict Long-Term Offspring Outcomes. J. Clin Child Adolesc. 2014;0(0):1-14.

25. Orgilés M, Samper M. El impacto del divorcio en la calidad de vida de los niños de 8 a 12 años de edad en la provincia de Alicante. Gac Sanit 2011;25(6):490-4.

26. Martínez-Pampliega A, Sanz M, Iraurgi J, Iriarte L. Impacto de la ruptura matrimonial en el bienestar físico y psicológico de los hijos. Síntesis de los resultados de una línea de investigación. La Revue du REDIF. 2009;2:7-18.

27. Kleinsorge C, Covitz L. Impact of divorce on children: Developmental considerations. Pediatr Rev. 2012;33(4):47-54.

28. Shulman S, Zlotnik A, Shachar-Shapira L, Connolly J, Bohr Y. Adolescent Daughters' Romantic Competence: The Role of Divorce, Quality of Parenting, and Maternal Romantic History. J Youth Adolescence. 2012;41:593-606.

29. Fergusson D, McLeod G, and Horwood J. Parental separation/divorce in childhood and partnership outcomes at age 30. J Child Psychol Psychiatr. 2014;55:352-60.

30. Roberts A, McLaughlin A, Conron J, Koenen C. Adulthood stressors, history of childhood adversity, and risk of perpetration of intimate partner violence. Am J Prev Med. 2011;40:128-38.

31. Sassler S, Cunningham A, Lichter D. Intergenerational patterns of union formation and relationship quality. J Fam Issues. 2009;30:757-86.

32. Orgilés M, Carratalá E, Espada. Perceived quality of the parental relationship and divorce effects on sexualbehaviour in Spanish adolescents. Psychol Health Med. 2015;20(1):8-17.

33. Huurre T, Junkkari H, Aro H. Long-term psychosocial effects of parental divorce: a follow-up study from adolescence to 
adulthood. Eur Arch Psychiatry Clin Neurosci. 2006;256(4):256-63.

34. Clark B. Supporting the mental health of children and youth of separating parents. Paediatr Child Health. 2013; 18(7):373-7.

35. Sandler I, Wolchik S, Winslow E, Mahrer N, Moran J, Weinstock D. Quality of maternal and paternal parenting following separation and divorce. En: Kuehnle K y Drozd L. eds. Parenting plan evaluations: Applied research for the family courts, New York: Oxford University Press, 2012; 85-122.

36. Lamela D, Figueiredo B. Coparenting after marital dissolution and children's mental health: a systematic review, (fecha de acceso: 06-06-2016). Disponible en: http://dx.doi.org/10.1016/j. jped.2015.09.011

37. Booth A, Scott M, King V. Father residence and adolescent problem behaviour: Are youth always better off in two-parent families? J. Fam. Issues. 2010;31:585-605

38. Bastaits K, Ponnet K, Mortelmans D. Parenting of Divorced Fathers and the Association with Children's Self-Esteem. J Youth Asolescence. 2012;41:1643-56.

39. Carlsund A., Eriksson U, Löfstedt P, Sellström E. Risk behaviour in Swedish adolescents: is shared physical custory after divorce a risk or protective factor? Eur J Public Health. 2013; 23:1,3-8.

40. Fransson E, Turunen J, Hjern A, Östberg V, Bergström M. Psychological complaints among children in joint physical custody and other family types: Considering parental factors. Scand J Public Health. 2016;44(2):177-830

41. Kalil A, Mogstad M, Rege M, Votruba M. Divorced Fathers' Proximity and Children's Long-Run Outcomes: Evidence From Norwegian Registry Data. Demography. 2011;48:1005-102.

42. Maier H. Tres teorías sobre el desarrollo del niño: Erikson, Piaget y Sears. En: Amorrortu editores. Buenos Aires: 2001; 19-61.

43. Kline M. Parenting plans following separation/divorce: Developmental considerations. Encyclopedia on Early Childhood Development: Divorce and separation. 2011:1-6. (Fecha de acceso 20-10-15]. Disponible en: http://www. child-encyclopedia.com/Pages/PDF/ divorce_and_separation.pdf

44. Roizblatt A. Divorcio y Familia: Antes, durante y después. Santiago, Editorial Ril, 2014.

45. McIntosh J, Wells Y, Smyth B, Long C. Child-focused and child-inclusive divorce mediation: comparative outcomes from a prospective study of postseparation adjustment. Fam Court Rev. 2008;46(1):105-24.

46. Alba-Fisch M. Collaborative Divorce: An Effort to Reduce the Damage of Divorce. J Clin Psychol. 2016;72(5):444-57. 